\title{
EVALUATION OF THE ZYGOMATIC BUTTRESS AS A DONOR SITE FOR RIDGE AUGMENTATION PRIOR TO IMPLANT INSERTION IN THE ESTHETIC ZONE
}

\author{
Mohamed M. Solaiman ${ }^{1} B D S$, Ahmed S. EL Mahallawy ${ }^{2} P h D$, Nagy E. Hassan ${ }^{2} P h D$
}

\begin{abstract}
INTRODUCTION: Traumatic loss of teeth in the esthetic zone commonly results in significant loss of buccal bone. This leads to reduced esthetics, problems with phonetics and reduction in function. Single tooth replacement has become an indication for implant-based restoration. In case of lack of bone volume the need of surgical reconstruction of the alveolar ridge is warranted. Several bone grafting techniques have been described to ensure sufficient bone volume for implantation.

OBJECTIVES: Evaluation of using the zygomatic buttress as an intraoral bone harvesting donor site for pre-implant grafting.

MATERIALS AND METHODS: Twelve patients were selected with limited alveolar ridge defect in the esthetic zone that needs bone grafting procedure prior to dental implants. Patients were treated using a 2-stage technique where bone blocks harvested from the zygomatic buttress region were placed as onlay grafts and fixed with osteosynthesis micro screws. After 4 months of healing, screws were removed for implant placement

RESULTS: Harvesting of 12 bone blocks were performed for all patients indicating a success rate of $100 \%$ for the zygomatic buttress area as a donor site. Final rehabilitation with dental implants was possible in 11 of 12 patients, yielding a success rate of 91.6\%. Three patients (25\%) had postoperative complications at the donor site and one patient (8.3\%) at the recipient site. The mean value of bone width pre-operatively was $3.64 \pm .48 \mathrm{~mm}$ which increased to $5.47 \pm .57 \mathrm{~mm}$ post-operatively, the increase in mean value of bone width was statistically significant $(\mathrm{p}<0.001)$.
\end{abstract}

CONCLUSIONS: Harvesting of intraoral bone blocks from the zygomatic buttress region is an effective and safe method to treat localized alveolar ridge defect before implant placement.

KEYWORDS: Zygomatic buttress, autogenous bone graft, dental implant, esthetic zone.

1-B.D.S (2011), Faculty of Dentistry, Alexandria University, Egypt

2-Professor of Oral and Maxillofacial Surgery, Faculty of Dentistry, Alexandria University, Egypt

\section{INTRODUCTION}

The reconstruction of skeletal deficiencies presents a challenging problem to the oral and maxillofacial surgeon and surgical community. Such defects in the facial skeleton can be the result of trauma, infection, congenital defects, cranio-facial syndromes, severe periodontitis or tumor resection (1).

Alveolar ridge resorption after tooth loss is a common phenomenon. After tooth extraction the alveolar ridge decreases in width and height very rapidly as much as $50 \%$ loss in width during the first year, two-thirds of which occurs in the initial 3 month (2).

In the reconstructive process there is often a need to create new bone. A solely prosthetic approach to management of alveolar bone loss frequently leads to esthetic and/or functional compromises (3).

The use of dental implants for the reconstruction of edentulous jaws has been a progressively growing treatment modality since the late 1970's. Brånemark and coworkers (4) published their first follow-up report of osseointegrated implants in the treatment of the edentulous jaw in the year 1977.

A prerequisite for the use of oral implants is a sufficient amount of bone to fully cover the implant and to allow the implant to support a fixed prosthetic restoration. Even a minor lack of bone either horizontal or vertical may cause a significant problem. A narrow or buccally concave alveolar ridge may result in exposed threads at the alveolar crest or at bone fenestrations (5).

Many allografts and alloplastic materials have been used as bone graft substitutes but autogenous corticocancellous bone grafts has still remained the gold standard for the reconstruction of alveolar bone. Bone grafting of the resorbed alveolus for dental implants was employed by Breine and Brånemark (6).

Researches in the field of oral and maxillofacial autografting surgery have produced new surgical techniques and bone harvesting donor sites for bone augmentation in deficient sites. The goal of these studies is the same to reduce complications and post-operative morbidity and to minimize the economic costs of the treatment $(7,8)$.

The use of extra-oral bone harvesting donor sites such as the anterior and posterior iliac crest is still the standard when large reconstructions are performed in the maxillomandibular region for example after tumor surgery or in dental implant treatment to totally edentulous jaws (7-10). However, the current trend when implant surgery is done to partially edentulous resorbed dento- alveolar ridges is to harvest bone from an intra-oral donor site. The most commonly utilized intra-oral bone harvesting donor sites in dental implant related surgery are the mandibular symphysis and ramus $(9,11,12)$.

The first reports of intra-oral bone harvesting and bone grafting for dental implants were published at the beginning of the 1990's by Jensen \& Sindet-Pedersen (9) and Misch et al. (11) Most of these reports highlighted the intra-oral harvesting sites as having convenient surgical access. The ischemic time of the bone graft has reported to be short.

Furthermore, since both the donor and recipient sites are intra-oral, there was no morbidity from a second surgical site. The morbidity associated with intra-oral donor sites was also found to be lower compared to extra-oral donor sites and the use of a trans-oral approach does not cause visible scarring. One major disadvantage of intra-oral bone harvesting was the limited amount of available bone $(9,11,13-16)$. 
The zygomatic buttress is a strong bony pillar that provides pressure absorption and transduction in the facial skeleton. This donor site has the great advantage that no muscles have to be detached and the bony structure in this area is especially strong (17).

The aim of this study was to evaluate the possibility of using the zygomatic buttress as an intraoral donor site of autogenous block bone graft for reconstruction of limited alveolar ridge defect in the esthetic zone.

\section{MATERIALS AND METHODS}

\section{Informed Consent:}

Appropriate institutional ethical clearance from the Faculty Ethical Committee and written informed consent from the patients were obtained. All patients were informed about the aim of the designed study.

\section{Patient Selection and Evaluation:}

This study was conducted on 12 patients having missing maxillary anterior teeth in the esthetic zone with alveolar ridge defect indicated for ridge augmentation prior to implant placement. Patients selected from the outpatient clinic of the Oral and Maxillofacial Surgery Department, Faculty of Dentistry, Alexandria University.

The inclusion criteria of this study were: patients having limited edentulous space in esthetic zone of the anterior maxilla with inadequate bone quantity for implant placement, adequate oral hygiene and patients psychologically accepting to participate in the study. While the exclusion criteria were: patients need grafting of bone defects caused by tumor resections, osteo-radionecrosis, or bisphosphonate-associated osteonecrosis, relevant uncontrolled systemic and/or metabolic diseases, immunosuppressive and/or autoimmune diseases, history of radiotherapy or chemotherapy, heavy smokers and parafunctional habits.

\section{Materials}

Titanium osteosynthesis micro screws: NORMED titanium osteosynthesis system (CNormed Zimmer Biomet Holdings, Indiana USA). It supplies micro screws with diameter of $1.2 \mathrm{~mm} / 1.7 \mathrm{~mm} / 2.3 \mathrm{~mm} / 2.7 \mathrm{~mm}$, and length range of $3-19 \mathrm{~mm}$.

Implant system: Super Line \& NR implant systems of dentium dental implants (DentiumTM dental implant, Seoul, Korea) is a titanium dental implant system consisting of mount free tapered fixture design with biological connection for load distribution to minimize micromovement and marginal bone loss. The conical hex connection between implant fixture and abutment interface ensure hermetic sealing, all implant diameters share the same internal hex. Implants are sandblasted, large grit and acid etching (SLA) Surface treatment, and double threaded with increased thread height to increase primary stability. It offers bone level implants in the size range of $3.2-7 \mathrm{~mm}$ diameter with 7 - 14 mm length.

\section{Pre-operative phase}

Preoperative clinical examination was performed for all patients. Patients' data were collected; name, gender and age, medical and dental history was taken and the oral mucosa of the edentulous area was examined for color, texture, firmness and thickness. Also, preoperative evaluation for all patients by cone beam computerized tomography (CBCT) to evaluate the residual ridge height, length and width, and for virtual treatment planning as shown in (Figure 1).

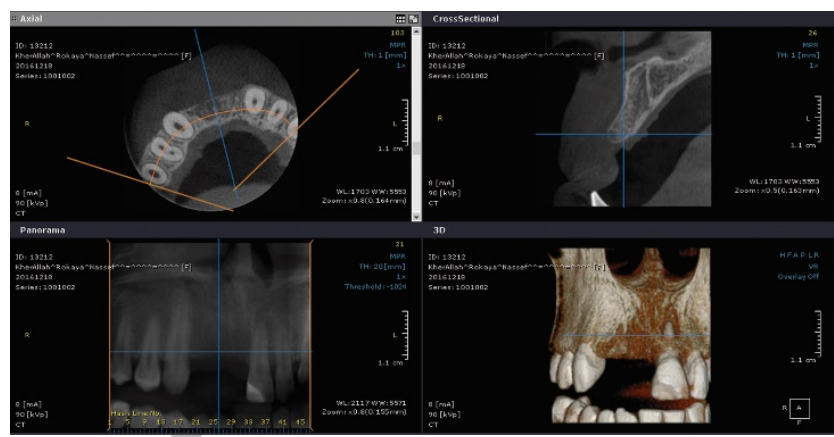

Figure 1: The preoperative CBCT showing missing maxillary RT $1^{\text {st }}, 2^{\text {nd }}$ incisors.

\section{Surgical Procedure}

All patients were operated under local anesthesia using articaine hydrochloride 4\% and levonordefrin (Septanest; Septodont, France). The oral mucosa was painted using povidone-Iodine solution 10\% (Betadine, antiseptic solution, SEDICO, Egypt) to render the surgical field free from microorganisms. Patients were treated using a 2-stage technique. The zygomatic buttress was exposed through a vestibular full thickness mucoperiosteal flap extending over the premolar-molar area, which reflected by periosteal elevator. The osteotomy was carried out with a small rotary bur outlining the bone block according to the virtual treatment plan measurements of the ridge defect and predetermined bone block size. A thin chisel was gently tapped along the entire length of the osteotomy line with care to maintain this parallel to the lateral surface of the zygomatic bone to guard against perforation of the maxillary sinus membrane (Figure 2).
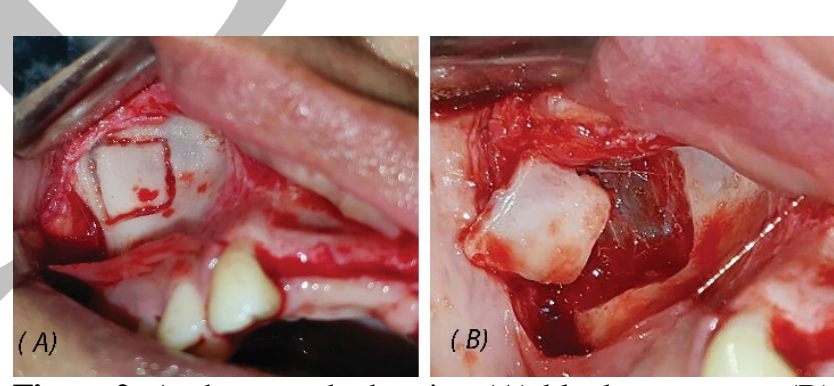

Figure 2: A photograph showing (A) block osteotomy (B) block elevation.

The zygomatic buttress bone graft was fixed with titanium osteosynthesis micro screws (NORMED, Zimmer Biomet Holdings, Indiana USA). Periosteal releasing incisions were made where necessary to achieve easy suturing without tension to the flap. The second stage after a minimum of 4 months of healing, raising a mucoperiosteal flap, removal of the screws and implant insertion (Figure 3 and 4).

All patients were advised to apply cold packs extra orally intermittently and avoid hot food on the first day, apply hot packs on the second day, avoid eating hard food at the surgical site, chlorhexidine mouth wash (Hexitol: the Arab Drug Company, Cairo, A.R.) was started on the 2nd post-operative day 3 times daily for 2 weeks. Also patients were instructed to avoid sneezing, nose blowing or other actions that might create high intranasal pressure or vacuum, avoid drinking with straws for a week and not to 
wear any prosthesis over the surgical site for at least one week after surgery to reduce the risk of wound dehiscence.

Postoperative administration of a broad spectrum antibiotic Amoxicillin 875 mg + Clavulanic acid 125 mg tablets (Augmentin $1 \mathrm{gm}$ Smithkline Beecham Pharmaceutical Co., Brentford, England) every 12 hours for 7 days to avoid post-operative infection. Non-steroidal antiinflammatory analgesic in the form of Diclofenac potassium $50 \mathrm{mg}$ tablets (Cataflam $50 \mathrm{mg}$ tablets, Novartis Pharma AG, Basle, Switzerland) every 8 hours for 7-10 days to avoid the possibility of inflammation, edema and pain. Ephedrine nasal drops (Otrivin spray/nasal Drops 10 ml, Novartis Pharma AG, Basle, Switzerland) 3-5 times daily for 5 days.
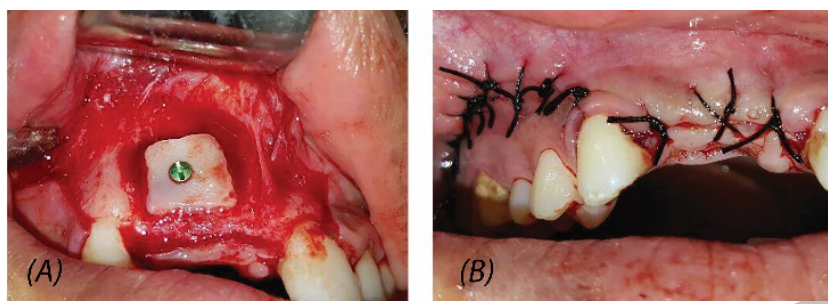

Figure 3: A photograph showing (A) graft in place (B) suturing.
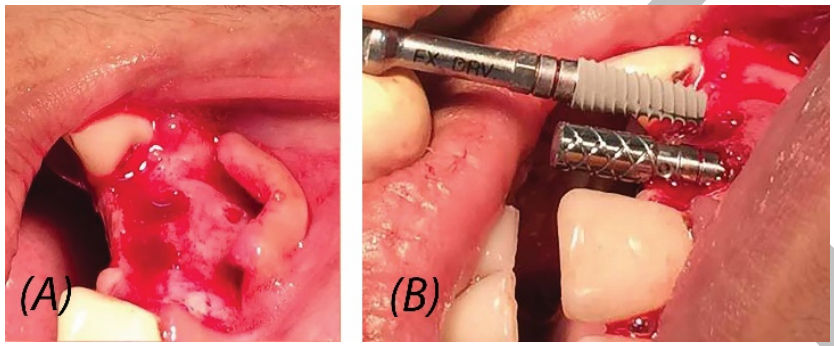

Figure 4: A photograph showing (A) implant osteotomy (B) implant placement.

\section{Postoperative evaluation}

All patients were examined the day after surgery then weekly for the first month postoperatively, then on intervals of 1, 2 and 4 months postoperatively for any complications at the donor and recipient sites such as pain, hemorrhage, dehiscence, infection or sensory disturbances. Pain and discomfort were examined using Numeric Pain Rating Scale (NPRS) $(18,19)$. Edema was evaluated by its ability to pit according to visual descriptor scale(VDS) (20).

Immediate evaluation of the graft fixed with titanium osteosynthesis micro screw in place by periapical $\mathrm{x}$-ray. CBCT was performed 4 months postoperatively for all patients to measure bone density and the amount of horizontal width gained, as shown in (figure 5 and 6). CBCT measurements were performed using On Diamond 3D App-DBM software system (version 1.0.9, Cybermed, Korea) where the bone width could be measured in $\mathrm{mm}$ and through which the bone density is calculated directly in HU. Virtual treatment plan was done for placement of implants using On Demand 3D ${ }^{\mathrm{TM}}$.

Statistical analysis of the data (21)

Data were fed to the computer and analyzed using IBM SPSS software (Package version 20.0. IBM Corporation, 1 New Orchard Road, Armonk, New York, United States). Quantitative data were described using range (minimum and maximum), mean, standard deviation and median. Qualitative data were described using number and percent.
Paired Samples T-Test was used to analyze the significance between the different stages. To compare between the different periods Related Samples Wilcoxon Signed Rank Test was applied. Significance of the obtained results was judged at the $5 \%$ level.

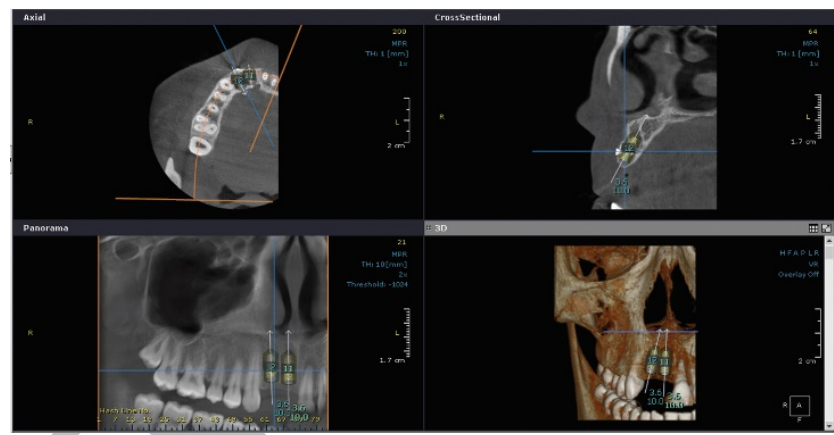

Figure 5: Postoperative virtual treatment plan for implant placement.

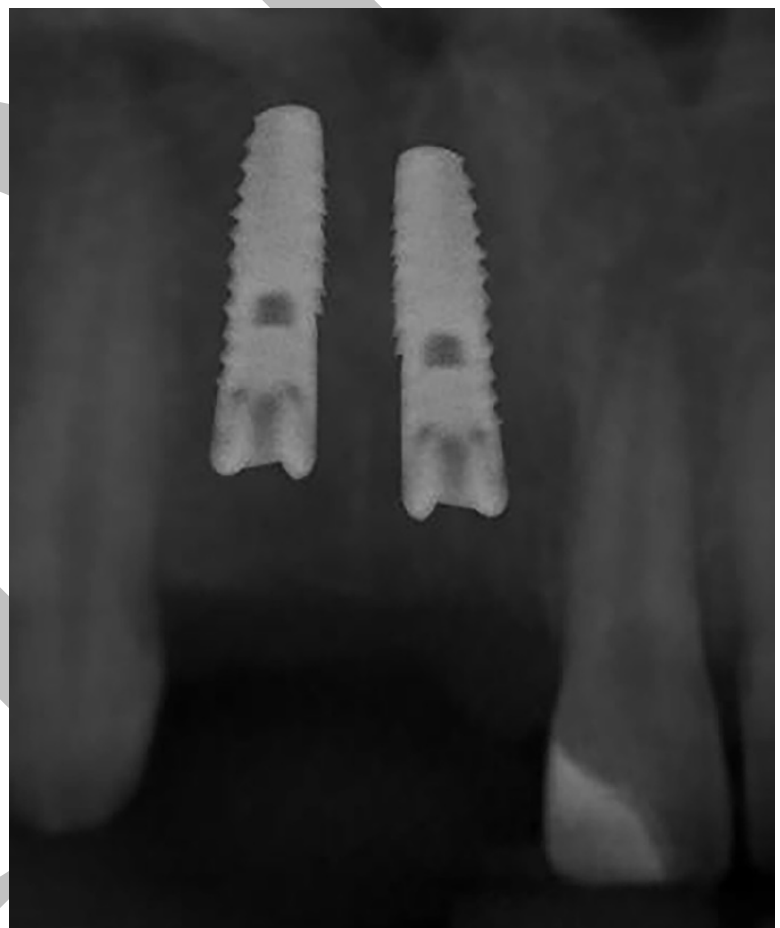

Figure 6: a photograph showing post implant placement $\mathrm{x}$-rays

\section{RESULTS}

In this study, a total of twelve zygomatic buttress bone block were harvested from twelve patients. . The selected patients were 6 males and 6 females, and their age ranged from 22-45 years with a mean age of 32.3 years. All patients presented with adequate bone height and length but with horizontal bone defect of the buccal plate of bone in the esthetic area of the anterior maxilla, the mean width of the alveolar ridge was $3.64 \pm .48 \mathrm{~mm}$ (Range: 2.56 - $3.95 \mathrm{~mm}$ ). Seven patients had single missed tooth, four patients had two missed teeth and one patient had three missed teeth. 2 patients of the study sample grafted for two teeth defect while 10 patients grafted as single tooth defect. Regarding the number of dental implant placement a total of 15 implants were placed for 11 patients in the size range of 3 $3.5 \mathrm{~mm}$ diameter with $10-12 \mathrm{~mm}$ length. 


\section{Clinical evaluation}

\section{Pain}

Pain was evaluated daily for the first week and after 2 weeks using numeric pain rating scale (NPRS) from 0 to 10 ("0" is pain free and "10" is unbearable pain). After surgery, four patients experienced mild pain (NPRS $=3$ ), seven patients experienced moderate pain (NPRS $=4-5)$ and one patient experienced severe pain (NPRS=7) at surgical site for the first day. Gradually decrease in pain intensity was observed, by the end of the first week no pain was recorded for patients.

\section{Edema}

After surgery, eleven patients expressed mild edema (+2) and one patient expressed moderate edema (+3) which gradually decreased along time during the first week postoperatively, that all patients recorded a trace edema $(+1)$ at the fourth day except one patient recorded mild edema (+2). By the end of the first week, edema totally subsided.

\section{Post-operative complications}

Three patients (25\%) had postoperative complications at the donor site, 2 of them had sinus membrane perforation during graft harvesting with no postoperative symptoms of sinusitis or oro-antral communication, the third had bleeding with hematoma formation along with wound dehiscence, that completely healed by the 2nd week postoperative leaving mild ecchymosis which disappeared by the 4th week postoperative. In the recipient site one patient $(8.3 \%)$ had excessive bone graft resorption leaving inadequate bone width for implant placement; this patient had a second grafting procedure for additional ridge augmentation.

\section{Radiographic evaluation}

Postoperative CBCT was performed for all patients after a minimum of four months healing period of the graft for:

1. Assessment of the amount of horizontal width gained, in the preoperative phase, the mean horizontal bone width value was $3.64 \pm .48 \mathrm{~mm}$ with a minimum recorded value of $2.56 \mathrm{~mm}$ and a maximum recorded value of $3.85 \mathrm{~mm}$. In the postoperative phase, the mean horizontal bone width value was $5.47 \pm .57 \mathrm{~mm}$ with a minimum recorded value of $3.94 \mathrm{~mm}$ and a maximum recorded value of 5.87 mm (table 1). These differences were statistically significant $(\mathrm{p}<0.001)$.

2. Assessment of the bone density of the newly formed bone, in the preoperative phase, the mean bone density value was $807.02 \pm 146.53 \mathrm{HU}$ with a minimum recorded value $484.46 \mathrm{HU}$ and a maximum recorded value of 955.01 HU. In the postoperative phase, the mean bone density was $838.33 \pm 191.89 \mathrm{HU}$ with a minimum recorded value of 503.67 and a maximum recorded value of $10066.76 \mathrm{HU}$ (table 2). These differences were statistically insignificant $(\mathrm{p}>0.001)$.
Table1: Comparison between preoperative and postoperative bone width $(\mathrm{mm})(\mathrm{n}=12)$.

\begin{tabular}{|l|l|l|l|}
\hline $\begin{array}{l}\text { Bone width } \\
\text { (mm) }\end{array}$ & Preoperative & Postoperative \\
\hline $\begin{array}{l}\text { Min. - Max. } \\
\begin{array}{l}\text { Mean } \pm \text { SD. } \\
\text { Median }\end{array}\end{array}$ & $2.56-3.95$ & & $3.94-5.87$ \\
& 3.83 & $5.47 \pm .57$ \\
\hline $\begin{array}{l}\text { \% of } \\
\text { change }\end{array}$ & $51.04 \pm 8.30$ & 5.71 \\
\hline Sig. & $0.002^{*}$ & \\
\hline
\end{tabular}

Sig. bet. Periods was done using Related Samples Wilcoxon Signed Rank Test.

*Statistically significant at $\mathrm{p} \leq 0.05$.

Table 2: Comparison between preoperative and postoperative mean bone density in HU $(n=12)$.

\begin{tabular}{|c|c|c|}
\hline Bone density in HU & Preoperative & Postoperative \\
\hline Min. - Max. & $484.46-$ & $503.67-1066.76$ \\
Mean \pm SD. & 955.01 & $838.33 \pm 191.89$ \\
Median & $807.01 \pm$ & 877.30 \\
& 146.53 & \\
& 839.53 & \\
\hline \% of change & \multicolumn{2}{|c|}{$8.82 \pm 8.15$} \\
\hline Sig. & \multicolumn{2}{|c}{$0.388^{*}$} \\
\hline \multicolumn{2}{|c|}{} \\
DISCUSSION
\end{tabular}

Augmentation of alveolar bone defects before dental implant insertion has been discussed recently in several clinical studies. Alveolar crest defects have been particularly scrutinized because they are the limiting factor in optimal implant positioning. If the bony recipient site does not fulfill the later implant-based prosthodontic requirements, failure of the whole treatment is likely to occur. Different augmentation techniques and materials have been investigated (17).

The aim of this study was to report the clinical results, rate of complications, and bone graft success after alveolar ridge augmentation in partially edentulous areas in the esthetic zone prior to implant placement, using bone blocks from the zygomatic buttress region to be implanted into small- to medium-sized alveolar defects. The evaluation of survival rate of the inserted implants was not the aim of this study. There is no other similar study in the current literature that describes the amount of width gained and bone density after augmentation with zygomatic buttress bone block graft.

In this study a total of 12 zygomatic buttress bone block grafts in 12 patients were performed in our clinic department. All patients were treated using a 2-stage technique; after 4 to 6 months of healing, the titanium osteosynthesis micro screws were removed and fifteen implants were placed. Sakkas A, et al. (22) in his study found that cases of delays of more than 6 months, the lack of stimulation sometimes led to severe graft resorption so that the osteosynthesis screws were transmucosally visible.

Patients were selected free from systemic diseases because that may complicate the surgical procedure or the healing process of the bone graft and implant placement procedure as advocated by Bolender (23), Dhanrajani and Al-Rafee (24) and Moy et al (25). Also in the year 1997 Balshi and Wolifinger (26) reported that patients were selected free from para-functional habits such as bruxism and clenching, because the magnitude of the forces are high, 
the duration of the forces are extensive and the direction of the forces are more horizontal than axial to the implants.

Heavy smokers were also excluded from this study. This followed the studies of Holahan et al (27) and Clementini et al (28), where they concluded that exposure to smoking has a harmful effect on the peri-implant bone loss that eventually lead to implant failure.

In addition, patients were selected free from any sinus pathology as recommended by $\mathrm{Li}$ and Wang (29) and Torretta et al (30). They recommended preoperative evaluation to rule-out any existing pathological condition that will certainly reduce the risk of mucus and bacteria contaminating the surgical field, along with postoperative complications that can be life threatening due to the proximity of maxillary sinus to vital structures.

In this study CBCT was performed for all patients preoperatively. Corresponding to the studies conducted by Cassetta et al (31) and Bornstein et al (32), they reported that the use of CBCT in implant dentistry vary from preoperative analysis regarding specific anatomic considerations, site development using grafts and treatment planning to postoperative evaluation. Along with, lower radiation dose, reduced costs and the relative grey density values of CBCT images making it a useful substitute for computerized tomography (CT) (33).

In this study the ability of harvesting bone blocks of different sizes adequate for alveolar ridge defect augmentation from the zygomatic buttress area were performed for all patients indicating a success rate of $100 \%$ for the zygomatic buttress area as a donor site for autografting procedures. Final rehabilitation with dental implants was possible in 11 of 12 patients, yielding a success rate of $91.6 \%$, despite the number of complications; rehabilitation with oral implants was not possible in only one case of all bone grafting procedures, as fifteen dental implants were placed, that seven patients received single dental implant and four patients had two dental implants. In this study, the majority of patients experienced mild to moderate pain, well tolerated with oral analgesics and gradually relieved by time without any discomfort to the patients. Patients suffered from postoperative edema which considered normal sequelae after surgical trauma, by the third day postoperatively half of the edematous swelling has been resolved.

Graft loss and graft removal were defined as failure. Bleeding, wound dehiscence, infection with pus, sensory disturbance, and perforations of the maxillary sinus membrane were defined as complications. According to our criteria, 3 patients (25\%) had postoperative complications at the donor site and one patient (8.3\%) at the recipient site. Throughout, 8 (66.6\%) of the bone grafts were successful and $4(33.3 \%)$ had complications such as incision-line dehiscence, sinus membrane perforation, or excessive graft resorption.

No major complications were observed regarding donor sites apart from 2 patients who had sinus membrane perforation, one of them considered as iatrogenic perforation that had happened after graft removal during wound preparation for closure. No postoperative symptoms of sinusitis or oro-antral communication were developed for both patients. The third patient with donor site complication had bleeding with hematoma formation along with wound dehiscence fortunately at the donor site only, that completely healed by the 2nd week postoperative leaving mild ecchymosis which disappeared by the 4th week postoperative.

In the recipient sites, except for minor complications such as excessive bone graft resorption in only one case was observed, because of patient traveling; a long standing graft healing resulted in excessive resorption of the bone graft yielding inadequate bone width for implant placement, this patient had a second grafting procedure for additional ridge augmentation.

This is in accordance with Sakkas A, et al. (22) in his study of the outcomes and complications of zygomatic buttress bone graft, yielding a failure rate of $0.01 \%$ which coincided with this study that presented a successful harvesting procedure. Sakkas A, et al., demonstrated that (82.3\%) of the bone grafts were successful and (17.6\%) had complications, he stated that $(3.5 \%)$ had postoperative complications at the donor site (17.8\%) at the recipient site. This study presented a success rate of (66.6\%) and a complication rate of (33.3\%).

This study concluded a success rate of $91.6 \%$ regarding final rehabilitation with dental implants, along with Sakkas A, et al., study that recorded $98.2 \%$ of implant placement success. Scabbia et al., (34) and Levin et al., (35) stated that smokers experienced a high failure rate and more postoperative complications than non smokers. An association between zygomatic buttress block bone grafting complications and smoking habits was also found in the study of Sakkas A, et al., as there was a high incidence of smoking among the patients in the study (66\%), in contrast this study had only 2 light smoking patients (16.6\%) free from any postoperative complications.

In this study, the mean original bone width was $3.64 \pm$ $.48 \mathrm{~mm}$ pre-operatively, while the mean postoperative bone width was $5.47 \pm .57 \mathrm{~mm}$ with mean bone width difference $1.82 \pm .16 \mathrm{~mm}$. The increase in horizontal bone width was found to be statistically significant $(\mathrm{p}<0.001)$.

The preoperative mean bone density value was $807.02 \pm$ 146.53 HU, while the postoperative mean bone density was $838.33 \pm 191.89 \mathrm{HU}$ with mean bone density difference $71.61 \pm 70.06$. This difference was found to be statistically insignificant $(p>0.001)$ which indicates that the zygomatic buttress bone yields bone grafts with similar bone density as the alveolar bone.

According to Gellrich et al., (17) the comparative advantages of the zygomatic buttress region as a donor site are as the following, this donor site offers easy access with excellent visibility, and yields good quality bone of the correct morphology. The zygomatic buttress is a strong bony pillar providing pressure absorption and transduction in the facial skeleton. This donor site has the great advantage that no muscles have to be detached, and the bony structure in this area is especially strong. In the case of an otherwise non traumatized facial skeleton, a bone graft of $1.5-2 \mathrm{~cm} 2$ taken from the caudal zygomatic buttress zone will not compromise the strength of the lateral midface frame.

With the described technique, it is possible to harvest approximately $0.5-1 \mathrm{~mL}$ of bone without causing damage to surrounding tissues. This amount of bone is sufficient to cover exposed implant threads and is adequate to reconstruct alveolar defects in the maxilla of a width of between 1 and 2 teeth. The convex cross-section of the bone graft is ideal for the reconstruction of alveolar projection loss in the anterior and posterior maxillary zone. The 
zygomatic buttress bone block graft patients appeared to have fewer difficulties in managing postoperative edema and pain. This clinical experience was also reported by Gellrich et al (17).

A limiting factor for zygomatic buttress bone graft is the mucous membrane of the adjacent maxillary sinus. Of all our patients undergoing this type of bone harvesting, a maxillary sinus perforation were observed in two patients. No postoperative sinusitis or oro-antral communication in the donor site was noted in any of these cases. However, the incidence of membrane perforation to the maxillary sinus does not significantly influence the success of this method of bone grafting, which makes bone harvesting with zygomatic buttress bone block graft a safe method prior to implant placement. Ideally, preoperatively, the patient should have a negative history of any sinus diseases.

Moreover, another limiting factor which is the close relationship to the infra-orbital foramen. However, direct visualization of the infra-orbital region allows nerve identification and preservation during bone graft harvesting.

Sakkas A, et al., (22) pointed out that less experienced surgeons tend not to reach the zygomatic buttress and therefore remove the graft from the laterally facial sinus wall, resulting in a thinner bone graft, almost always causing membrane perforation. Above of all, atrophy of the zygomatic area related to a syndrome or congenital abnormality, as well as previous surgery or trauma to the area, could be considered as contraindications to zygomatic buttress bone harvesting.

Because the amount of bone harvested from the zygoma is smaller than that from mandibular donor sites, this technique is best suited for situations in which only moderate amounts of bone are needed, especially when implant surgery is undertaken in the maxilla. The amount of bone that we harvested from this donor site was adequate to reconstruct alveolar defects of a width of between 1 and 2 teeth.

The cumulative treatment time is reduced due to the combined recipient and donor site approach for the grafting procedure, reducing the number of surgical procedures and sites, the pain medications required post-surgically and recovery time, resulting in reducing the total cost of patient treatment.

Finally, the complications documented in this study after autogenous bone harvesting from the zygomatic buttress did not influence significantly the success of the final dental rehabilitation. The compliance of patients during the entire surgical therapy was excellent, despite the need for second surgical treatments.

\section{CONCLUSION}

Harvesting of intraoral bone blocks from the zygomatic buttress region is an effective and safe method to treat localized alveolar ridge defect before implant placement. The postoperative discomfort after graft harvesting is comparable to postsurgical complaints after dento-alveolar surgery and the complication rate is very low, above all the total outcome is very good. This method of bone grafting represents an excellent alternative for augmentation of limited anterior and posterior maxillary defects.

\section{CONFLICT OF INTEREST}

The authors declare that they have no conflicts of interest.

\section{REFERENCES}

1. Chiapasco M, Zaniboni M, Boisco M. Augmentation procedures for the rehabilitation of deficient edentulous ridges with oral implants. Clin Oral Implants Res. 2006;136-59.

2. Toscano N, Shumaker N, Holtzclaw D. The art of block grafting: A review of the surgical protocol for reconstruction of alveolar ridge deficiency. J Implant Adv Clin Dent. 2010;2-957.

3. Williamson RA. Rehabilitation of the resorbed maxilla and mandible using autogenous bone grafts and osseointegrated implants. Int J Oral Maxillofac Implants. 1996;476-88.

4. Brånemark PI, Hansson BO, Adell R, Breine U, Lindström J, Hallén $\mathrm{O}$, et al. Osseointegrated implants in the treatment of the edentulous jaw. Experience from a 10year period. Scand J Plast Reconstr Surg Suppl. 1977;16:1-132.

5. Adell R, Lekholm U, Gröndahl K, Brånemark PI, Lindström J, Jacobsson M. Reconstruction of severely resorbed edentulous maxillae using osseointegrated fixtures in immediate autogenous bone grafts. Int J Oral Maxillofac Implants. 1990;5:233-46.

6. Breine U, Brånemark PI. Reconstruction of alveolar jaw bone. An experimental and clinical study of immediate and preformed autologous bone grafts in combination with osseointegrated implants. Scand J Plast Reconstr Surg. 1980;14:23-48.

7. Cordaro L, Amadé DS, Cordaro M. Clinical results of alveolar ridge augmentation with mandibular block bone grafts in partially edentulous patients prior to implant placement. Clin Oral Implants Res. 2002 Feb;13:103-11.

8. Cordaro L. Bilateral simultaneous augmentation of the maxillary sinus floor with particulated mandible. Report of a technique and preliminary results. Clin Oral Implants Res. 2003 Apr 1;14:201-6.

9. Jensen J, Sindet-Pedersen S. Autogenous mandibular bone grafts and osseointegrated implants for reconstruction of the severely atrophied maxilla: a preliminary report. J Oral Maxillofac Surg Off J Am Assoc Oral Maxillofac Surg. 1991 Dec;49:1277-87.

10. Misch CM. Comparison of intraoral donor sites for onlay grafting prior to implant placement. Int J Oral Maxillofac Implants. 1997 Dec;12:767-76.

11. Misch CM, Misch CE, Resnik RR, Ismail YH. Reconstruction of maxillary alveolar defects with mandibular symphysis grafts for dental implants: a preliminary procedural report. Int J Oral Maxillofac Implants. 1992;7:360-6.

12. Misch CM. Ridge augmentation using mandibular ramus bone grafts for the placement of dental implants: presentation of a technique. Pract Periodontics Aesthetic Dent PPAD. 1996;8:127-135.

13. Sindet-Pedersen S, Enemark H. Mandibular bone grafts for reconstruction of alveolar clefts. J Oral Maxillofac Surg Off J Am Assoc Oral Maxillofac Surg. 1988;46:533-7.

14. Sindet-Pedersen S, Enemark H. Reconstruction of alveolar clefts with mandibular or iliac crest bone grafts: a comparative study. J Oral Maxillofac Surg Off J Am Assoc Oral Maxillofac Surg. 1990;48:554-558.

15. ten Bruggenkate CM, Kraaijenhagen HA, van der Kwast WA, Krekeler G, Oosterbeek HS. Autogenous 
maxillary bone grafts in conjunction with placement of I.T.I. endosseous implants. A preliminary report. Int J Oral Maxillofac Surg. 1992;21:81-4.

16. Jensen J, Sindet-Pedersen S, Oliver AJ. Varying treatment strategies for reconstruction of maxillary atrophy with implants: results in 98 patients. J Oral Maxillofac Surg Off J Am Assoc Oral Maxillofac Surg. 1994;52:210-216.

17. Gellrich N-C, Held U, Schoen R, Pailing T, Schramm A, Bormann K-H. Alveolar Zygomatic Buttress: A New Donor Site for Limited Preimplant Augmentation Procedures. J Oral Maxillofac Surg. 2007;65:275-80.

18. Broome ME. Measurement of Pain: Self-Report Strategies. J Pediatr Oncol Nurs. 1991;8:131-3.

19. McCaffery M, Beebe A, Latham J, Ball D. Pain : clinical manual for nursing practice. UK ed. St.Louis, MO : Mosby; 1994.

20. Yokobe J, Kitahara M, Matsushima M, Uezono S. Preference for different anchor descriptors on visual analogue scales among Japanese patients with chronic pain. PloS One. 2014;9-98.

21. Kotz S BN, Read CB, Vidakovic B. Encyclopedia of statistical sciences. In N.J: Wiley-Interscience; 2006;3359.

22. Sakkas A, Schramm A, Karsten W, Gellrich N-C, Wilde F. A clinical study of the outcomes and complications associated with zygomatic buttress block bone graft for limited preimplant augmentation procedures. J Cranio-Maxillofac Surg. 2016;44:249-56.

23. Bolender CL. Indications and contraindications for different types of implant therapy. J Dent Educ. 1988;52:757-9.

24. Dhanrajani PJ, Al-Rafee MA. Single-tooth implant restorations: a retrospective study. Implant Dent. 2005;14:125-30.

25. Moy PK, Medina D, Shetty V, Aghaloo TL. Dental implant failure rates and associated risk factors. Int J Oral Maxillofac Implants. 2005;20:569-77.

26. Balshi TJ, Wolfinger GJ. Two-implant-supported single molar replacement: interdental space requirements and comparison to alternative options. Int J Periodontics Restorative Dent. 1997;17:426-35.

27. Holahan CM, Koka S, Kennel KA, Weaver AL, Assad DA, Regennitter FJ, et al. Effect of osteoporotic status on the survival of titanium dental implants. Int $\mathrm{J}$ Oral Maxillofac Implants. 2008;23:905-10.

28. Clementini M, Rossetti PHO, Penarrocha D, Micarelli C, Bonachela WC, Canullo L. Systemic risk factors for peri-implant bone loss: a systematic review and metaanalysis. Int J Oral Maxillofac Surg. 2014;43:323-34.

29. Li J, Wang H-L. Common implant-related advanced bone grafting complications: classification, etiology, and management. Implant Dent. 2008;17:389-401.

30. Torretta S, Mantovani M, Testori T, Cappadona M, Pignataro L. Importance of ENT assessment in stratifying candidates for sinus floor elevation: a prospective clinical study. Clin Oral Implants Res. 2013;24:57-62.

31. Cassetta M, Sofan AA, Altieri F, Barbato E. Evaluation of alveolar cortical bone thickness and density for orthodontic mini-implant placement. J Clin Exp Dent. 2013;5:245-252.

32. Bornstein MM, Scarfe WC, Vaughn VM, Jacobs R. Cone beam computed tomography in implant dentistry: a systematic review focusing on guidelines, indications, and radiation dose risks. Int $\mathrm{J}$ Oral Maxillofac Implants. 2014;29:55-77.

33. Mello LA de, Garcia RR, Leles JLR, Leles CR, Silva MAGS. Impact of cone-beam computed tomography on implant planning and on prediction of implant size. Braz Oral Res. 2014;28:46-53.

34. Scabbia A, Cho KS, Sigurdsson TJ, Kim CK, Trombelli L. Cigarette smoking negatively affects healing response following flap debridement surgery. J Periodontol. 2001;72:43-9.

35. Levin L, Herzberg R, Dolev E, Schwartz-Arad D. Smoking and complications of onlay bone grafts and sinus lift operations. Int $\mathrm{J}$ Oral Maxillofac Implants. 2004;19:369-73. 